https://doi.org/10.48009/2_iis_2005_182-189

\title{
CULTURAL IMPACT ON THE DESIGN OF E-COMMERCE WEBSITES: PART I - SITE FORMAT AND LAYOUT
}

\author{
Bruce W. N. Lo, University of Wisconsin-Eau Claire, lobw@uwec.edu \\ Panqun Gong, Southern Cross University, gongq@scu.edu.au
}

\begin{abstract}
How to "think globally, act locally" is a challenge facing many e-commerce companies that want to extend their business to other parts of the globe. To address the cultural dimension of this problem, this paper examines a selection of leading e-commerce websites from the United States and China to determine what impact cultural differences have on the format and layout of their design. The implications of cultural influences on website design are discussed.
\end{abstract}

Keywords: culture, e-commerce, globalization, website design, web localization

\section{INTRODUCTION}

It has been estimated that the world-wide online populations will reach 1.1 billion in 2005 , and the majority of them are from non-English speaking language zones [6, 10]. More interestingly, for the first time, the combined Sino-Japanese online language group (29\%) will exceed the English language group (27\%). Figure 1 provides a detailed breakdown of the top 10 language groups in the global online population.

Although the majority of ecommerce websites are in English, companies that want to extend their businesses to the global market can no longer ignore the non-English sector. The challenge is how to create an online presence that will incorporate local culture to increase its appeal to the targeted audience.
Figure 1. Estimated 2005 Online Population Language Groups [6]

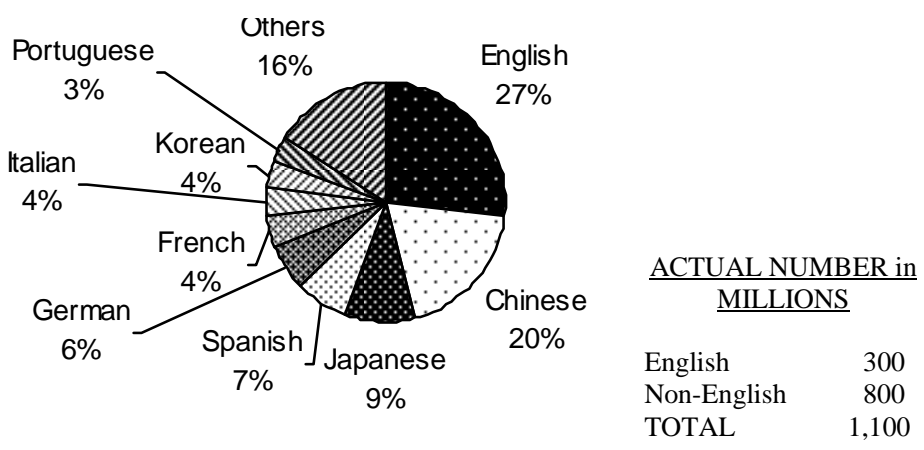

With respect to the globalization influence of e-commerce, there are two opposing points of view [11]. Some argued that the dominance of transnational e-commerce giants created a converging trend of standardization in web communication and contents. While others argued that there are evidences of cultural sensitivity in thematic appearance, interactivity, and content. Therefore localization of website is critical to business success. Although recent studies appear to lean towards the latter, the issue is by no means closed. The aim of this study is to seek further clarification on the debate by looking at what are actually practiced by e-commerce web masters.

One can approach the study of cultural influences on e-commerce websites in two ways. One is to examine the attitude or behavior of e-commerce consumers. This measures the expectation of 
e-commerce clients. The other is to examine what website designers do to cater for culturally diverse clienteles. This provides a snapshot of the current status of e-commerce websites. Both have certain degrees of validity and can shed light on the subject under consideration. This paper reports the findings of a preliminary study where the second approach was used. This study is part of an ongoing research on Culture and Internet", where both approaches are being used.

Most popular discussions on the cultural differences on E-commerce focus on language and customs issues [5]. In this investigation we analyze a selection of 100 leading E-commerce websites (50 from the US and 50 from China) to determine the extent of cultural impact on the site design along three dimensions: format, content, and interactivity. The purpose is to discover whether there are any underlying principles which may be learned, and which may help website designers to address issues facing E-commerce in different cultural environments. The ecommerce websites were chosen from these two countries because English and Chinese are currently the two largest language groups of online population (27\% and $20 \%$ respectively), and also because they are most likely to show observable cultural differences (compare to, say, two European language groups). Because national culture is susceptible to the globalization influence of Internet, it is important to use subjects that are least likely marred by background noises.

The research questions that this paper attempts to address are:

- Do the design features of this selection of e-commerce websites reveal a trend in global standardization or show a greater degree of localization?

- Are there evidences of cultural impact on the format of e-commerce website design in the set of selected sites?

- Are there evidences of cultural factors impacting on the layout of the web pages in this collection of e-commerce websites?

In our research study, two further questions were investigated: (a) the cultural impact on website content, and (b) the cultural impact on website interactions. Due to space limitation, the findings to these two questions will be considered in a forthcoming paper.

\section{CONCEPTS AND RELATED STUDIES}

The concept of culture is very complex. Many anthropologists believe that it involves at least three interrelated components: what people think, what they do, and what they produce. Perhaps the most widely known study on cultural differences came from Hofstede [8], who proposed that at least half of the variance between different countries could be explained by just 5 variables: power distance (PDI), individualism vs collectivism (IDV), masculinity vs femininity (MAS), uncertainty avoidance (UAI), and long-term orientation (LTO). The implications of cultural influence on user interface and web design have been taken up by a number of authors [2]. It is not possible to explore all aspects of culture in the present study. Furthermore culture is dynamic and constantly evolving. Our discussion here shall focus on those characteristics that can be readily observed on the selected set of websites. What observables to include is not a simple question. Our decision is arrived at after a careful examination of recent studies on the subject.

An early paper by Jarvenpaa et al. [8] considered the question of trust for Internet store consumers across a variety of culture. Another early, and probably most widely quoted paper by Aoki [1], compared the cultural difference in e-commerce between US and Japan. This paper laid the foundation for website content analysis for several subsequent studies, including the 
consideration of payment systems as a form of website interaction. In their paper on website navigation (which is an aspect of website interactivity), Luna et al. [9] introduced the concept of cultural congruity which they claimed will enhance site visitors' e-commerce experience. Next came two studies comparing e-commerce sites in the US and China [2, 12], and another study comparing US and Hong Kong [3]. The second paper by Singh et al. [12] went into some detail to analyze Hofstede's variables and their implications for web design. More recently Singh et al. [11] raised the issue of the two opposing trends of "to localize or to standardize" in a 5-nation study involving Italy, India, Netherlands, Spain, and Switzerland.

Based on these observations, the website characteristics we had chosen for this research include color usage, page layout, site content, and the nature of website interactions. Each of these will be examined to determine whether there is any evidence of cultural sensitivity among the selected websites. As stated earlier, only the results for the first two are reported here. The findings relating to the last two characteristics will be reported separately.

- Color usage includes the choice of color for webpage background, foreground, frame, image, hyperlink and logo.

- Page layout includes components of the webpage: such as banners, animation, splashwindows, rollover-graph/text, buttons, icons, white space and website navigation models.

- Site content refers to (a) website type: portal site, retail site, service site, entertainment site etc; and (b) site strategy: advertising, promotional, informational, or entertainment.

- Interactivity includes such tasks as payment system, feedback, e-mail contact, phone, online knowledge support, online form, FAQ, search, site map.

\section{RESEARCH METHOD}

The 100 e-commerce websites chosen for our study consist of 50 from China and 50 from the US. The 50 Chinese sites were the most popular sites reported by China Internet Network Information Center, CNNIC [4] eliminating those that are obviously not true e-commerce sites. The CNNIC ranking criteria are based on customer survey, web expert evaluation, regional ranking references, and market research. The original list was obtained in 2002. We recognize that the list changes over time and have attempted to monitor it over time. We determine that the changes do not significantly alter our findings and conclusion in this study. The original set of top 50 US sites was obtained from Jupiter Media Metrix. Jupiter was acquired by Neilsen NetRating in 2002. A current list of most visited sites may be found in Ranking.com [13].

Each website was examined with respect to the four characteristics mentioned above, namely, color usage, page layout, site content, and interactivity. The frequencies of occurrence were counted, categorized, and tabulated. Where applicable, Chi-square analyses were performed to test for significant differences in frequency distributions between the countries. The results on color usage and page layout, are presented in table and graphic forms in the next section.

\section{RESULTS AND ANALYSIS}

Space limitation necessitates that the findings of our investigation be presented in an abridged form. Those interested in additional details about the results may contact the authors directly. We shall present the results in four subsections, corresponding to each of the four site characteristics. 


\section{Color Usage}

Color plays a very important role in our perception which is influenced by our cultural background. To see how site designers take this into consideration, we counted the number of times a color is used in the home page of a website originating from each country. The frequencies were determined for the nine common colors (red, orange, yellow green, blue, purple, black, white, gray) plus an "other" category. The usage was tabulated for 6 types of webpage objects: foreground, background, image, frame, hyperlinks, and logos. Table 1 presents these results. The last row gives the total color counts for all webpage objects.

Table 1. Color Usage in Background, Foreground, Frame, Images, Hyperlinks and Logos

\begin{tabular}{llllllllllllll} 
& Cntry & Wht & Blk & Blu & Red & Grn & Ylw & Org & Ppl & Pnk & Gry & Oth & TOT \\
\hline Bkgnd & US & 37 & 2 & 4 & 0 & 1 & 4 & 0 & 0 & 0 & 2 & 0 & 50 \\
& CN & 44 & 0 & 1 & 0 & 1 & 2 & 0 & 0 & 0 & 2 & 0 & 50 \\
\hline \multirow{2}{*}{ Frgnd } & US & 10 & 17 & 32 & 5 & 1 & 15 & 11 & 1 & 0 & 8 & 3 & 103 \\
& CN & 8 & 24 & 31 & 23 & 7 & 23 & 5 & 0 & 0 & 17 & 3 & 141 \\
\hline Frame & US & 22 & 6 & 17 & 1 & 2 & 17 & 4 & 0 & 0 & 10 & 5 & 84 \\
& CN & 17 & 4 & 27 & 10 & 8 & 25 & 8 & 0 & 1 & 22 & 5 & 127 \\
\hline Image & US & 7 & 19 & 22 & 10 & 14 & 16 & 5 & 1 & 2 & 3 & 0 & 99 \\
& CN & 15 & 19 & 43 & 42 & 41 & 38 & 30 & 8 & 17 & 2 & 6 & 261 \\
\hline Hplnk & US & 8 & 15 & 28 & 11 & 6 & 10 & 7 & 1 & 1 & 3 & 2 & 92 \\
& CN & 4 & 30 & 31 & 31 & 10 & 17 & 8 & 1 & 0 & 7 & 0 & 138 \\
\hline Logo & US & 16 & 16 & 17 & 16 & 7 & 9 & 4 & 0 & 1 & 3 & 0 & 89 \\
& CN & 5 & 31 & 21 & 28 & 7 & 17 & 6 & 0 & 1 & 5 & 0 & 121 \\
\hline \multirow{2}{*}{ TOTAL } & US & 100 & 75 & 120 & 43 & 31 & 71 & 31 & 3 & 4 & 29 & 10 & 517 \\
color & CN & 93 & 108 & 154 & 134 & 74 & 122 & 57 & 9 & 19 & 55 & 17 & 839 \\
\hline
\end{tabular}

The first impression from this table is that Chinese websites make use of a greater number of colors per page than their US counterparts. To examine this in more detail, we graphed the "percentage" color usage for each of the webpage objects. Due to space limitation, only 4 of the 7 figures are presented below: background, foreground, hyperlinks, and total color usage.

The follow observation may be made:

- The predominant color for background is white for both countries (Figure 2). Background color usage here appears to show some trend for standardization.

- In terms of the foreground color usage (Figure 3), blue, black, and yellow are the three leading colors in both countries. But it would be difficult not to notice the prominence of red as a foreground color in Chinese sites.

- In terms of total color usage, Figure 5 shows that the dominant colors for US are blue (23\%), white (19\%), Black (15\%) and Yellow (14\%); and for Chinese are blue (18\%), red $(16 \%)$, yellow (15\%), and black (13\%). Blue is the most frequently used for both countries. But the prominence of red and the relative de-emphasis of white in Chinese sites are clearly evident. Traditionally Chinese associate red with good luck, wealth, and marriage (e.g., bridal dress), while white has been associated with death and mourning.

- The US sites show a clear trend of using blue color for hyperlinks (Figure 4). But in the Chinese sites, all three colors, black, blue and red hyperlinks are equally likely. 
- With respect to logo colors (graph not shown), black and red are the clear favorites for Chinese sites while white, black, blue, and red have equal frequencies on the US sites.
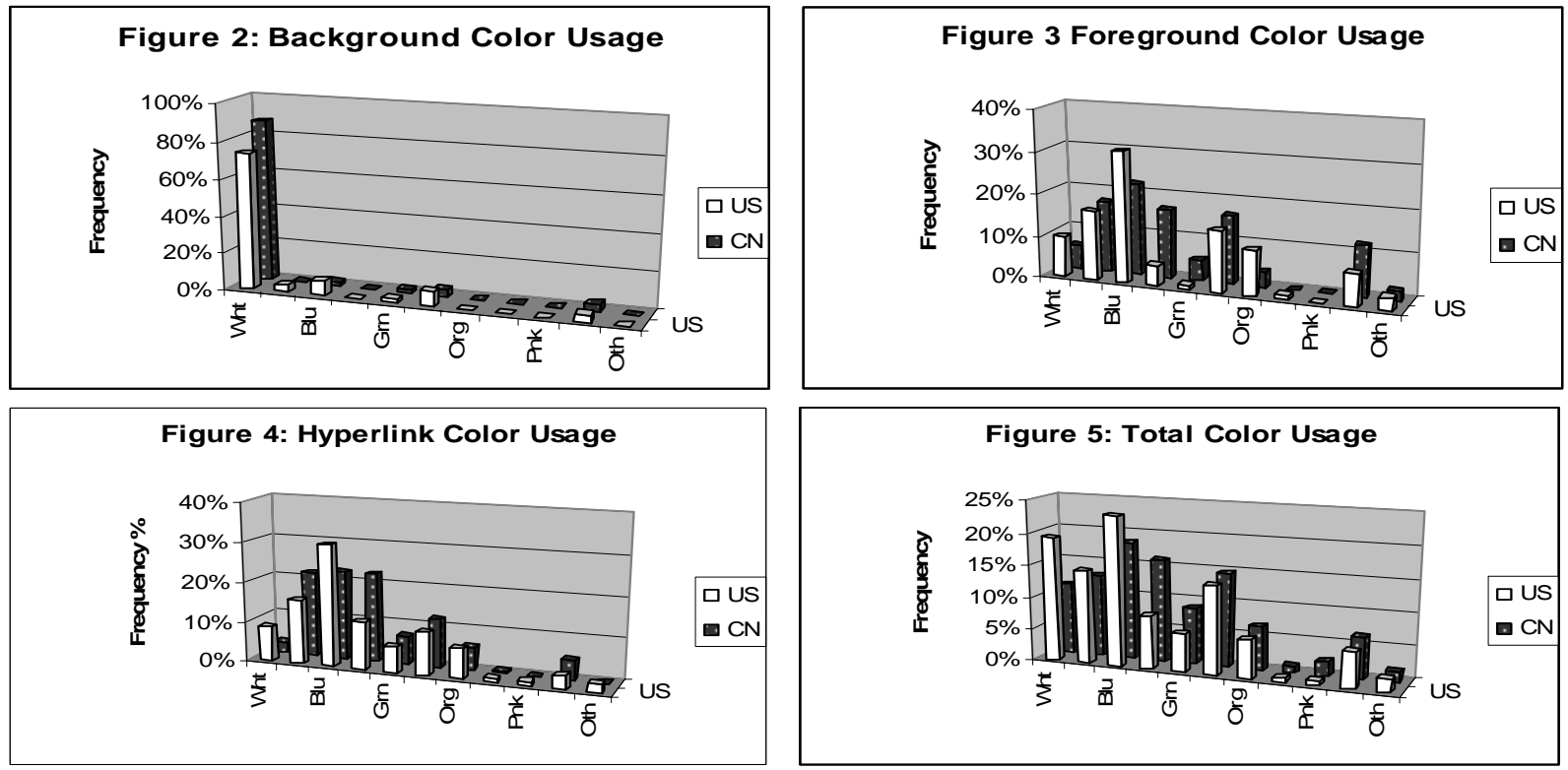

To determine whether there is a significant difference between the two countries in color usage, Chi-square test was applied to the total color usage data. Test statistics was calculated to be $\chi^{2}=$ 41.09 , with d.f. $=(2-1)(10-1)=9$. The critical value $\chi_{c}^{2}=16.92$. Since $\chi^{2}>\chi_{c}^{2}$, we reject the null hypothesis of no difference and conclude that the color usage patterns are significantly different.

\section{Webpage Layout}

Webpage layout affects the look and feel of a website. The variables to be considered here are: the banners usage, animation, rollover-graph/text, buttons, icons, splash windows, and the use of "white space". In addition to the above, we also consider navigation models of the website. In terms of the direction of navigation, 5 navigation models are possible: left-oriented, rightoriented, top-oriented, bottom-oriented, and center-oriented. In terms of the appearance of navigation buttons, it can be text-based or it can be GUI-based.

Table 2 gives the results of this investigation. It was found that the number of active banners per page is 11 times higher and active animations are 9 times higher in the Chinese sites than the US sites. The same can be said about the use of rollover graphs/text and frames. However, there was no significant difference in the use of splash windows. In contrast, the US made use of white space a lot more than their Chinese counterparts. However, the Chinese sites used a greater number of buttons per page, while the US sites used a greater number of icons per page.

Table 2. Page Layout Characteristics

\begin{tabular}{llllllll} 
Type of Layout & US & CN & Unit & Type of Layout & US & CN & Unit \\
\hline Active Banner & 0.10 & 1.14 & Per page & Frames & 5.5 & 9.3 & Per website \\
Active Animation & 0.52 & 4.92 & & White Space & 41 & 19 & Area \\
Rollover Graph/Text & $1 / 1$ & $17 / 13$ & Actual counts & Buttons & 7.3 & 12.8 & Per page \\
Splash Windows & 15 & 12 & & Icons & 1.20 & 0.72 &
\end{tabular}


Generally speaking, Chinese sites are more active (that is, having plenty of active banners and animation) and use a greater variety (and density) of layout elements, but with less white space. As a result they appear to be more cluttered. However, the "squareness" of Chinese characters helps to maintain a clear separation of distinctive web elements and orderly navigation.

The next two figures report on the use of navigation models. Figure 6 shows that, US sites favor GUI-based navigation buttons, while Chinese sites favor text-based navigation buttons. As to the direction of navigation, Figure 7 is even more interesting. The Chinese sites clearly favor the "top-oriented" navigation model, while the US usage is roughly equal among left-, top- and center-oriented navigation models. In a sense this is not surprising, as traditionally Chinese writings are read from top to bottom and right to left. However, the right to left navigation was not supported by the Chinese websites.
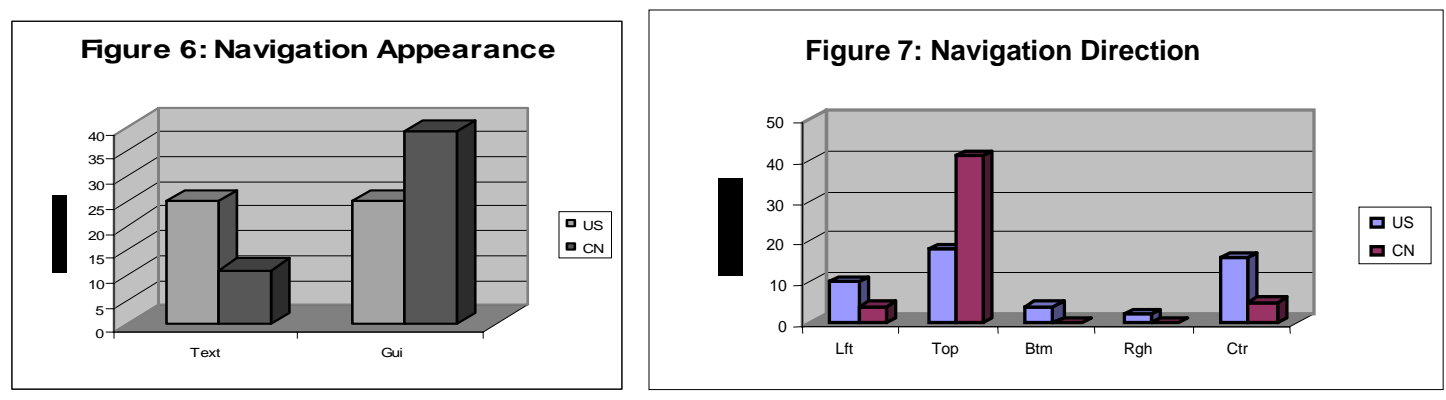

\section{DISCUSSION}

Can these findings be related to Hofstede's cultural dimensions? We venture to offer the following discussion. According to Hofstede [7], China is a country characterized by high power distance, collectivism, masculinity, and uncertainty avoidance. The US is characterized by high individualism, higher masculinity, low power distance and uncertainty avoidance.

High power distance and collectivism usually result in adherence to standards and norms dictated by the authority. One of the early entries to China's e-commerce world is Yahoo, which enjoyed huge popular success. The Chinese Yahoo site employs the top-oriented \& text-based navigation model. This model was also used by two other leading Chinese sites: sina.com and sohu.com. Their success was clearly recognized, becoming a symbolic figure of authority in Chinese web design. Subsequent entries to the Chinese e-commerce market tend to imitate their approach using the same navigation model (top-oriented \& text-based). This may account for the observed results in Figure 7. On the other hand, US is rated higher in individualism. Thus we find a greater variety of navigation styles and site design approaches among the US sites.

Another observation relates to the high uncertainty avoidance in Chinese culture. One of the major challenges in e-commerce is to build up customer trust [8]. This is particularly so for Chinese e-commerce website designers, because of the higher cultural "uncertainty avoidance" of their audience. It appears that a design element that designers have used to counteract this is to make use of lots of active webpage elements, such as animation, banners, rollover graphics \& texts, and buttons. These active elements are designed to booster the confidence and trust of website visitors towards the products/services offered by the e-commerce sites. The activeness also attracts larger groups of site visitors, reinforcing trust through the collectivism factor.

No discussion is complete without a consideration of the limitations of this study. As mentioned earlier, culture is very complex. It is important to recognize that what was considered here is a 
rather narrow view of culture. The collection of top e-commerce sites are changing all the time. Therefore this study is essentially a "snap shot" of what were out there. The analysis itself can be influenced by subjective bias also, as we count and classify. Therefore the results should be interpreted with care. In many case the home pages rather than the entire website were analyzed, as the later approach is prohibitively large. Finally there is a complex interplay between technology and culture which may require more than a few pages of discussion to fully explore.

What can we learn from these results thus far? Firstly, there is some evidence on the emergence of a global e-commerce culture or some common standards, for example using "blue" color for hyperlinks and de-emphasizing (or abandonment) the right-to-left navigation model used in traditional Chinese writing. However, there is also clear evidence of the need for e-commerce website designers to consider the impact of local culture, for example the higher frequency of using red color in Chinese websites. Therefore the two opposing trends, to standardize and to localize, are real, and are constantly evolving in a dynamic state of balance and counterbalance. E-commerce site designers should not ignore the intricate interplay between these two trends.

\section{REFERENCES}

1. Aoki, K. (2000) Cultural Differences in E-Commerce: A comparison between the U.S. and Japan, First Monday, 5(11), November. http://www.firstmonday.org/issues/issue5_11/aoki/.

2. Bin, Q, Chen, S, \& Sun, S, (2003). Cultural Differences in E-Commerce: A comparison between US \& China, Journal of global information Management, 11(2) 48-55.

3. Chau, P, Cole M., Massey, A, Montoya-Weiss, M. \& O’Keefe, R. (2002) Cultural Difference in Online Behavior of Consumers. Communications of the ACM, 45(10) 138-143.

4. China Internet Network Information Center (2005) $15^{\text {th }}$ Statistical Survey Report on Internet Development in China; Available http://www.cnnic.cn/en/index/index.htm, accessed 2/20/05.

5. Dr Ecommerce, (2001) What are the key cross cultural differences, that will affect ecommerce web site development? http://www.jpb.com/drecommerce/answers/000340.html.

6. Global Reach, (2004). Global statistics on world online populations by languages. Available http://global-reach.biz/globstats/evol.html Accessed 10/5/04. (Website closed on 3/1/05).

7. Hofstede, G.. (1996). Cultures and Organizations: Software of the Mind. New York: McGraw-Hill. See also IBE website link: http://www.cyborlink.com/besite/hofstede.htm

8. Jarvenpaa, S, Tractinsky, N, \& Saarinen, L. (1999). Consumer Trust in an Internet Stores: A cross-cultural validation, Journal of Computer Mediated Communications, 5(2), December, at http://www.ascusc.org/jcmc/vol5/issue2/jarvenpaa.html.

9. Luna, D, Peracchio, L, \& de Juan, M, (2002), Cross-Cultural and Cognitive Aspects of Web Site Navigation, Journal of the Academy of Marketing Science, 30(4) 397-410.

10. Pastore, M. (2001). Global Internet Population Moves Away from US. Available http://www.clickz.com/stats/sectors/geographics/article.php/558061, Accessed 2/15/05.

11. Singh, N, Furrer, O, \& Ostinelli, M. (2004) To Localize or Standardize on the Web: empirical evidence from Italy, India, Netherlands, Spain, and Switzerland, Multinational Business Review, 12(1) 69-87.

12. Singh, N, Zhao H, \& Hu, X. (2003) Cultural Adaptation on the Web: A study of American companies and Chinese websites, Journal of Global Information Management, 11(3) 63-80.

13. Ranking.com, (2005) Site Ranking Report on http://www.trafficranking.com/mainframe.htm, Accessed 2/28/05; See also Neilsen NetRating, Top Rankings - Netview Usage Metrics on http://www.netratings.com/, Earlier statistics obtained from Jupiter Media Metrix on www.jmm.com, which merged with Neilsen NetRating in 2002. 